\title{
VIBRATION ANALYSIS OF THREE-LAYERED NANOBEAMS BASED ON NONLOCAL ELASTICITY THEORY
}

\author{
Nouha Kammoun, Hanen Jrad, Slim Bouaziz \\ Laboratory of Mechanical Modeling and Production (LA2MP), National School of Engineers of Sfax, Tunisia \\ e-mail:kamoun.noha@gmail.com; hanen.j@gmail.com; slim.bouaziz1@gmail.com
}

Mohamed Soula

Laboratory of Applied Mechanics and Engineering (LR-May-ENIT), National School of Engineers of Tunis, Tunisia e-mail: soulamed2003@yahoo.fr

Mohamed Haddar

Laboratory of Mechanical Modeling and Production (LA2MP), National School of Engineers of Sfax, Tunisia e-mail: mohamed.haddar@enis.rnu.tn

\begin{abstract}
In this paper, the first investigation on free vibration analysis of three-layered nanobeams with the shear effect incorporated in the mid-layer based on the nonlocal theory and both Euler Bernoulli and Timoshenko beams theories is presented. Hamilton's formulation is applied to derive governing equations and edge conditions. In order to solve differential equations of motions and to determine natural frequencies of the proposed three-layered nanobeams with different boundary conditions, the generalized differential quadrature (GDQM) is used. The effect of the nanoscale parameter on the natural frequencies and deflection modes shapes of the three layered-nanobeams is discussed. It appears that the nonlocal effect is important for the natural frequencies of the nanobeams. The results can be pertinent to the design and application of MEMS and NEMS.
\end{abstract}

Keywords: beams theories, nonlocal elasticity theory, vibration analysis, GDQ method

\section{Introduction}

In the last few years, scientific researchers have been focusing on nanotechnology and the resulting nano-materials which play key roles in many engineering devices at the nano-scale used in several applications including microactuators, microswtiches, biosensors, nanowires, nanoprobes, ultra-thin films and micro-and nano-electromechanical systems (MEMS and NEMS) studied in works of (Hung and Senturia, 1999; Li et al., 2003; Moser and Gijs, 2007; Pei et al., 2004; Najar et al., 2010). In fact, nano-materials have special mechanical, chemical, electrical, optical and electronic properties. Modeling and analysis of nanostructures including nanobeams, nanofils, carbon and boron-nirtide nanotubes, nanoribbons and nanoplates which are mostly applied MEMS and NEMS, and tracking their mechanical behavior can give truthful and promising results for designing such devices.

Although classical theories of linear and nonlinear vibration of strings and beams at macroscales are well established, the vibration behavior of structures at the nanoscale, which is significantly size dependent, is far from being well understood. In fact, experimental and molecular dynamics simulation results (Bauer et al., 2011) have shown that the small-scale effects in the analysis of mechanical properties of nano- and micro-structures cannot be neglected. Due to being scale-free, the classical continuum theory is unable to accurately detect the static and dynamic mechanical behavior of nano- and micro-structures. 
Nonlocal continuum theories of elasticity has found successful applications in nanomechanics including lattice dispersion of elastic waves, wave propagation in composites, dislocation mechanics, fracture mechanics (Peddieson et al., 2003). Nonlocal theories that have been studied in the literature include Eringen's nonlocal elasticity theory (Eringen, 1972), modified couple stress theory of Mindlin (1963), Koiter (1964), and Toupin (1964), and the strain gradient theory (Mindlin, 1965; Lam et al., 2003). Eringen's nonlocal elasticity can be classified into a differential nonlocal form or an integral nonlocal form. Detailed review of both forms is discussed by Lim (2010).

Numerous researchers have studied the mechanical behavior of nano-sized structures based on Eringen's nonlocal elastic theory. Analytical solutions for bending, buckling and vibration of beams using the Euler-Bernoulli, Timoshenko, Reddy, and Levinson beam theories have been developed by Reddy (2007). Analytical study of bending, buckling and vibration response of a Euler-Bernoulli nanobeam was proposed in the work of Thai (2012). Using a meshless method based on collocation with a radial basis, Roque et al. (2011) studied static bending, buckling and free vibration behavior of a Timoshenko nanobeam. Considering the perturbation method, free vibration, steady-state resonance and stability of a vibrating nanobeam subjected to a variable axial load was studied by Li et al. (2011). Moreover, the finite element method was used by Eltaher (2013) to solve the vibration problem of a Euler-Bernoulli nanobeam. Nonlinear vibration of nanobeams is reported in several works. Reddy (2010) reformulated classical shear deformation beam and plate theories taking into account the von Karman nonlinear strains. The nonlinear pull-in instability of a nanoswitch modeled as an Euler-Bernoulli nanobeam subjected to electrostatic and intermolecular forces and having different boundary conditions was investigated by Mousavi et al. (2013) using the differential quadrature method. Later, nonlinear finite element analysis of the Euler-Bernoulli and Timoshenko beam theories with the von Karman nonlinear strains and Eringen's nonlocal model was developed by Reddy and El-Borgi (2014).

The choice of a discretization process is indispensable for obtaining the number of resulting ordinary differential equations. Discretized models are time efficient and can be strongly employed to determine dynamics of systems subject to simple excitations with very small displacements about a given equilibrium point (Shkel, 2006). Finite element lumping of MEMS and NEMS, including complex geometry and using commercial softwares would provide more rigorous results. However, this discretization remains a heavy step in the design procedure, even when using automated size-reduction routines. Furthermore, the dynamic behavior cannot be totally inspected using these models. However, there are other discretization methods, such as the generalized differential quadrature method (GDQM), which approximate the original mechanism by a small number of ordinary differential equations. These techniques preserve the complexity of the system response, due to nonlinearities, in a parameterized model that is well suited for relatively complex geometries. Using this approach, the system dynamics can be precisely modeled using fewer degrees of freedom.

This paper makes the first attempt to investigate vibration of three-layered nanobeams incorporating the mid-layer shear effect based on Eringen's nonlocal theory as well as Euler Bernoulli and Timoshenko beam theories. The nonlocal nanobeam model is developed to capture the size effect in three-layered nanostructures. The governing equations and boundary conditions are derived by using Hamilton's principle. The generalized differential quadrature method (GDQM) is employed to discretize the governing equations which are then solved to obtain natural frequencies and mode shapes of three-layered nanobeams with different edge conditions. The influence of the nonlocal parameter on the vibration of the three-layered nanobeams incorporating the mid-layer shear effect are discussed. 


\section{Equations of motion and boundary conditions of the nonlocal three-layred nanobeam model}

The majority of existing works on nonlocal elasticity are pertaining to the analysis of single nanobeams: nanotubes (Wang et al., 2007; Reddy, 2007; Behera et al., 2014) and nanoribbons (Nazemnezhad et al., 2015). Though mechanical studies of nanobeams may include buckling and vibration of multiple-walled nanotubes and multilayer nanoribbons, the study of discrete multi-layered nanobeams has not been reported in literature.

Recently, Nazemnezhad et al. (2016) discussed nonlocal vibration of multi-layer graphene nanoribbons (MLGNRs) incorporating the interlayer shear effect. In fact, multilayer graphene nanoribbons (MLGNRs) are single layers of nanoribbons held together by weak van der Waals (vdWs) forces. According to Nilsson et al. (2008) and Hosseini Kordkheili et al. (2013), these weak interlayer vdWs bindings induce considerable changes in electrical and mechanical properties of MLGNRs (Nilsson et al., 2008) and (Hosseini Kordkheili et al., 2013) and, consequently, static and dynamic behavior of MLGNRs will change.

Based on the above discussion, in this paper an investigation is carried out to illustrate the small-scale effects in the behavior of a three-layered nanobeam incorporating the interlayer shear effect. The studied nanobeam is constructed of a thin elastic layer sandwiched between two identical elastic layers. The following general assumptions are made when developing governing differential equations of motion in free vibration of a three-layered nanobeam and associated boundary conditions:

- The theory of linear elasticity is applied to all displacements and strains.

- Transverse normal strains in the three layers are negligible.

- There is continuity of displacement at the interfaces between the layers.

Considering Cartesian coordinate system, Fig. 1 shows a three-layered nanobeam of length $L$. Each layer has its own geometric properties with a subscript $i$ denoting the layer number $(i=1$ for the top layer). Thus each layer has thickness $h_{i}$, width $b_{i}$ (so that area $A_{i}=h_{i} b_{i}$ ).

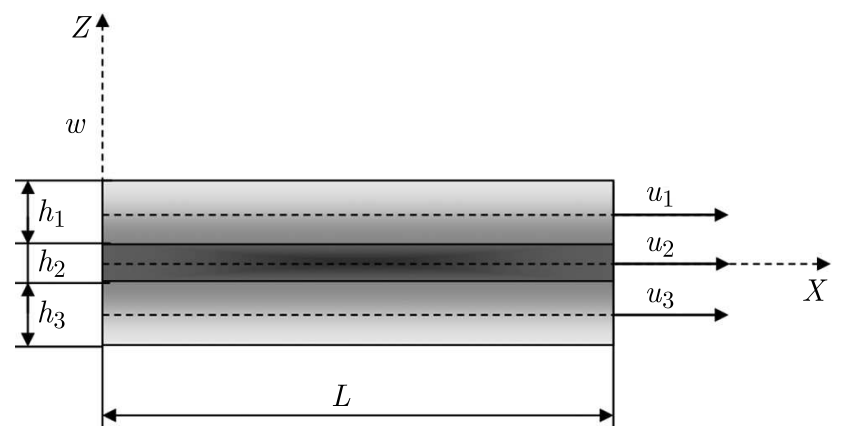

Fig. 1. Schematic configuration for a three-layered nanobeam incorporating the mid-layer shear effect

The system of displacements used is as follows. All three layers have common flexure in the $y$-direction with the flexural displacement denoted by $w$. The axial displacement (i.e. displacement in the $x$-direction) of the mid-plane of each layer is $u_{i}(i=1,2,3)$ which varies linearly through thickness, as shown in Fig. 1.

Assuming that the cross-section of each layer does not rotate so as to be normal to the common flexure, but it necessarily shears at the central layer, we propose to model the upper and lower layers by considering the Euler-Bernoulli beam formulation, and the central layer behavior is captured using the Timoshenko beam formulation taking into account small-scale effects. 
According to the Euler-Bernoulli beam theory, the displacement of an arbitrary point of layer (1) and layer (3) of the three-layered nanobeam along the $x$ and $z$ axes denoted by $u_{x i}(x, z, t)$ and $u_{z i}(x, z, t)$, respectively, are:

- for $\frac{h_{2}}{2} \leqslant z \leqslant \frac{h_{2}}{2}+h_{1}$

$$
\begin{array}{rlrl}
u_{z 1}(x, z, t) & =w_{1}(x, t) & u_{x 1}(x, z, t) & =u_{1}(x, t)-\left(z-\frac{h_{1}+h_{2}}{2}\right) w_{, x}(x, t) \\
- \text { for }-h_{3}-\frac{h_{2}}{2} \leqslant z \leqslant-\frac{h_{2}}{2} & & \\
u_{z 3}(x, z, t) & =w_{3}(x, t) & u_{x 3}(x, z, t) & =u_{3}(x, t)-\left(z+\frac{h_{3}+h_{2}}{2}\right) w_{, x}(x, t)
\end{array}
$$

where ',' symbolizes differentiation with respect to coordinates and $u_{1}, w_{1}$, and $u_{3}, w_{3}$ are the axial and transverse displacements of an arbitrary point located on the mid-axis of layer (1) and layer (3), respectively, and $t$ is time. It is further assumed for the transverse displacement that $u_{z 1}=u_{z 2}=u_{z 3}=w(x, t)$. For layer (2), the displacement is expressed using the Timoshenko beam theory for the shear effect taken into account

$$
u_{z 2}(x, z, t)=w(x, t) \quad u_{c}(x, z, t)=u_{2}(x, t)+z \phi(x, t) \quad-\frac{h_{2}}{2} \leqslant z \leqslant \frac{h_{2}}{2}
$$

where $\phi$ is rotation of the beam cross-section.

The strain-displacement equations of the three-layered nanobeam are given as follows

$$
\begin{array}{ll}
\varepsilon_{x x}^{(1)}=u_{1, x}-\left(z-\frac{h_{1}+h_{2}}{2}\right) w_{, x x} & \varepsilon_{x x}^{(2)}=u_{2, x}+z \phi_{, x} \\
\gamma_{x z}^{(2)}=w_{, x}+\phi & \varepsilon_{x x}^{(3)}=u_{3, x}-\left(z+\frac{h_{3}+h_{2}}{2}\right) w_{, x x}
\end{array}
$$

The strain energy $U$ of the three-layered nanobeam resulting from the advent of variation in the stresses with respect to the initial configuration is given by

$$
\begin{aligned}
U= & \frac{1}{2} \int_{0}^{L} \int_{A}\left(\sigma_{x x}^{(1)} \varepsilon_{x x}^{(1)}+\sigma_{x x}^{(2)} \varepsilon_{x x}^{(2)}+\sigma_{x z}^{(2)} \gamma_{x z}^{(2)}+\sigma_{x x}^{(3)} \varepsilon_{x x}^{(3)}\right) d A d x \\
= & \frac{1}{2} \int_{0}^{L}\left[N_{x}^{(1)} u_{1, x}+N_{x}^{(2)} u_{2, x}+N_{x}^{(3)} u_{3, x}-\left(M_{x}^{(1)}+M_{x}^{(3)}\right) w_{, x x}\right. \\
& \left.+M_{x}^{(2)} \phi_{, x}+Q\left(w_{, x}+\phi\right)\right] d x
\end{aligned}
$$

where $N_{x}^{(i)}, M_{x}^{(i)}$ and $Q$ are the normal resultant force, the bending moment and the transverse shear force for layer (i), respectively. They are obtained from

$$
\begin{array}{lc}
N_{x}^{(1)}=\int_{A} \sigma_{x x}^{(1)} d A=E A_{1} u_{1, x}=A_{11} u_{1, x} & N_{x}^{(2)}=\int_{A} \sigma_{x x}^{(2)} d A=E A_{2} u_{2, x}=A_{12} u_{2, x} \\
N_{x}^{(3)}=\int_{A} \sigma_{x x}^{(3)} d A=E A_{3} u_{3, x}=A_{13} u_{3, x} & M_{x}^{(1)}=\int_{A} \sigma_{x x}^{(1)} z^{(1)} d A=b_{1} D w_{, x x}=B_{11} w_{, x x} \\
M_{x}^{(3)}=\int_{A} \sigma_{x x}^{(3)} z^{(3)} d A=b_{3} D w_{, x x}=B_{13} w_{, x x} & M_{x}^{(2)}=\int_{A} \sigma_{x x}^{(2)} z d A=b_{2} D \phi_{, x}=B_{12} \phi_{, x} \\
Q_{x}=k_{s} \int_{A} \sigma_{x z}^{(2)} d A=k_{s} G A\left(\phi+w_{, x}\right)=k_{s} C_{12}\left(\phi+w_{, x}\right) &
\end{array}
$$


in which $A_{1}, A_{2}$ and $A_{3}$ are the cross section areas of layer (1), layer (2) and layer (3), respectively. $D$ is the bending rigidity of the three-layered nanobeam. $k_{s}$ is the shear correction factor depending on the shape of the cross section of the beam.

The kinetic energy $T$ can be expressed as

$$
T=\frac{1}{2} \int_{0}^{L} \int_{A} \rho\left(\dot{u}_{x i}^{2}+\dot{u}_{z i}^{2}\right) d A d x=\frac{1}{2} \int_{0}^{L}\left[I_{1}\left(\dot{u}_{1}^{2}+\dot{w}^{2}\right)+I_{2}\left(\dot{u}_{2}^{2}+\dot{w}^{2}\right)+I_{3} \dot{\phi}^{2}+I_{4}\left(\dot{u}_{3}^{2}+\dot{w}^{2}\right)\right] d x
$$

where '.' signifies differentiation with respect to time, $\left\{I_{1}, I_{2}, I_{3}, I_{4}\right\}=\rho\left\{A_{1}, A_{2}, I, A_{3}\right\}$ and $A_{i}=b_{i} h_{i}, I=b_{2} h_{2}^{3} / 12$.

Using Hamilton's principle $\left(\int_{0}^{t}(\delta T-\delta U) d t=0\right)$, the classical governing equations of the three-layered nanobeam are obtained as follows

$$
\begin{array}{lcc}
N_{x, x}^{(1)}=I_{1} \ddot{u}_{1}^{2} \quad N_{x, x}^{(2)}=I_{2} \ddot{u}_{2}^{2} \quad N_{x, x}^{(3)}=I_{4} \ddot{u}_{3}^{2} \quad M_{x, x x}^{(1)}=I_{1} \ddot{w}^{2} \\
M_{x, x}^{(2)}-Q_{x}=I_{3} \ddot{\phi}^{2} & Q_{x, x}=I_{2} \ddot{w}^{2} & M_{x, x x}^{(3)}=I_{4} \ddot{w}^{2}
\end{array}
$$

Considering a beam-type structure, thicknesses and widths are much smaller than its length. So that, for a beam with transverse motion in the $x z$-plane, we can assume that the nonlocal behavior is negligible in the thickness direction (Reddy, 2007). Then, nonlocal constitutive relation (2.3) can be approximated to a one-dimensional form expressed as in the following

$$
\sigma_{x x}-\left(e_{0} a\right)^{2} \frac{\partial^{2} \sigma_{x x}}{\partial x^{2}}=E \varepsilon_{x x} \quad \sigma_{x z}-\left(e_{0} a\right)^{2} \frac{\partial^{2} \sigma_{x z}}{\partial x^{2}}=G \gamma_{x z}
$$

where $E$ and $G$ are respectively the elastic modulus and shear modulus of the beam. $e_{0} a$ is the scale coefficient revealing the size effect on the response of the structures in the nanosize. $e_{0}$ is a material constant, and $a$ and $L$ are the internal and external characteristic lengths of the nanostructures, respectively. $\mu=e_{0} a / L$ is the nonlocal parameter.

To develop the nonlocal governing equations of motion of the three-layered nanobeam, it is necessary to obtain the nonlocal normal resultant force $N_{x}^{(i)}$, shear force $Q_{x}$ and bending moment $M_{x}^{(i)}$. From Eqs. (2.9) and (2.8), the nonlocal $N_{x}^{(i)}, Q_{x}$ and $M_{x}^{(i)}$ are defined as

$$
\begin{array}{ll}
N_{x}^{(1)}-\left(e_{0} a\right)^{2} N_{x, x x}^{(1)}=A_{11} u_{1, x} & N_{x}^{(2)}-\left(e_{0} a\right)^{2} N_{x, x x}^{(2)}=A_{12} u_{2, x} \\
N_{x}^{(3)}-\left(e_{0} a\right)^{2} N_{x, x x}^{(3)}=A_{13} u_{3, x} & M_{x}^{(1)}-\left(e_{0} a\right)^{2} M_{x, x x}^{(1)}=B_{11} w_{, x x} \\
M_{x}^{(3)}-\left(e_{0} a\right)^{2} M_{x, x x}^{(3)}=B_{13} w_{, x x} & M_{x}^{(2)}-\left(e_{0} a\right)^{2} M_{x, x x}^{(2)}=B_{12} \phi_{, x} \\
Q_{x}-\left(e_{0} a\right)^{2} Q_{x, x x}=k_{s} C_{12}\left(w_{, x}+\phi\right) &
\end{array}
$$

By substituting Eq. (2.10), into Eq. (2.8), the explicit expression of the nonlocal normal resultant force $N_{x}^{(i)}$, shear force $Q_{x}$ and bending moment $M_{x}^{(i)}$ can be written as

$$
\begin{array}{ll}
N_{x}^{(1)}=A_{11} u_{1, x}+\left(e_{0} a\right)^{2} I_{1} \ddot{u}_{1, x} & N_{x}^{(2)}=A_{12} u_{2, x}+\left(e_{0} a\right)^{2} I_{2} \ddot{u}_{2, x} \\
N_{x}^{(3)}=A_{13} u_{3, x}+\left(e_{0} a\right)^{2} I_{4} \ddot{u}_{3, x} & M_{x}^{(1)}=B_{11} w_{, x x}+\left(e_{0} a\right)^{2} I_{1} \ddot{w}_{, x} \\
M_{x}^{(3)}=B_{13} w_{, x x}+\left(e_{0} a\right)^{2} I_{4} \ddot{w}_{, x} & M_{x}^{(2)}=B_{12} \phi_{, x}+\left(e_{0} a\right)^{2} I_{3} \ddot{\phi}_{, x} \\
Q_{x}=k_{s} C_{12}\left(w_{, x}+\phi\right)+\left(e_{0} a\right)^{2} I_{2} \ddot{w}_{, x} &
\end{array}
$$

Then, the nonlocal governing equations of motion of the three-layered nanobeam can be expressed as

$$
\begin{array}{lc}
A_{11} u_{1, x x}=I_{1}\left(\ddot{u}_{1}-\left(e_{0} a\right)^{2} \ddot{u}_{1, x x}\right) & A_{12} u_{2, x x}=I_{2}\left(\ddot{u}_{2}-\left(e_{0} a\right)^{2} \ddot{u}_{2, x x}\right) \\
A_{13} u_{3, x x}=I_{4}\left(\ddot{u}_{3, x x}-\left(e_{0} a\right)^{2} \ddot{u}_{3, x x}\right) & B_{12} \phi_{, x x}-k_{s} C_{12}\left(w_{, x}+\phi\right)=I_{3}\left(\ddot{\phi}-\left(e_{0} a\right)^{2} \ddot{\phi}_{, x x}\right) \\
\left(B_{11}+B_{13}\right) w_{, x x x x}+k_{s} C_{12}\left(w_{, x x}+\phi_{, x}\right)=\left(I_{1}+I_{2}+I_{4}\right)\left(\ddot{w}-\left(e_{0} a\right)^{2} \ddot{w}_{, x x}\right)
\end{array}
$$




\section{Non-dimensional form of governing equations of motion of the nonlocal three-layred nanobeam model}

The non-dimensionalization procedure has important applications in the analysis of differential equations. In this part, all parameters of the governing equations of motion are changed to a dimensionless form in order to facilitate the resolution process. Considering the following dimensionless parameters as

$$
\begin{aligned}
& \xi=\frac{x}{L} \quad \eta=\frac{L}{H} \quad \mu=\frac{e_{0} a}{L} \quad \tau=\frac{t}{L} \sqrt{\frac{A_{t}}{I_{t}}} \quad A_{t}=A_{11}+A_{12}+A_{13} \\
& I_{t}=I_{1}+I_{2}+I_{4} \quad H=h_{1}+h_{2}+h_{3} \quad\left(U_{1}, U_{2}, U_{3}, W\right)=\left(\frac{u_{1}}{H}, \frac{u_{2}}{H}, \frac{u_{3}}{H}, \frac{w}{H}\right) \\
& \phi^{*}=\phi \quad C_{12}=\frac{c_{12}}{A_{12}} \quad\left(\bar{A}_{11}, \bar{A}_{12}, \bar{A}_{13}\right)=\left(\frac{A_{11}}{A_{11}}, \frac{A_{12}}{A_{12}}, \frac{A_{13}}{A_{13}}\right) \\
& \left(\bar{B}_{11}, \bar{B}_{12}, \bar{B}_{13}, \bar{C}_{12}\right)=\left(\frac{B_{11}}{A_{11} h_{1}^{2}}, \frac{B_{12}}{A_{12} h_{2}^{2}}, \frac{B_{13}}{A_{13} h_{3}^{2}}, \frac{C_{12}}{A_{12}}\right) \\
& \left(\bar{I}_{1}, \bar{I}_{2}, \bar{I}_{3}, \bar{I}_{4}\right)=\left(\frac{I_{1}}{I_{1}}, \frac{I_{2}}{I_{2}}, \frac{I_{3}}{I_{2} h_{2}^{2}}, \frac{I_{4}}{I_{4}}\right)
\end{aligned}
$$

Governing equation (2.12) can be rewritten taking into account the dimensionless parameters as

$$
\begin{array}{ll}
\bar{A}_{11} U_{1, \xi \xi}=\bar{I}_{1}\left(\ddot{U}_{1}-\mu^{2} \ddot{U}_{1, \xi \xi}\right) & \bar{A}_{12} U_{2, \xi \xi}=\bar{I}_{2}\left(\ddot{U}_{2}-\mu^{2} \ddot{U}_{2, \xi \xi}\right) \\
\bar{A}_{13} U_{3, \xi \xi}=\bar{I}_{4}\left(\ddot{U}_{3}-\mu^{2} \ddot{U}_{3, \xi \xi}\right) & \bar{B}_{12} \phi_{, \xi \xi}^{*}-k_{s} \bar{C}_{12} \eta\left(W_{, \xi}+\eta \phi^{*}\right)=\bar{I}_{3}\left(\ddot{\phi}^{*}-\mu^{2} \ddot{\phi}_{, \xi \xi}^{*}\right) \\
\left(\bar{B}_{11}+\bar{B}_{13}\right) W_{, \xi \xi \xi \xi}+k_{s} \bar{C}_{12}\left(W_{, \xi \xi}+\eta \phi_{, \xi}^{*}\right)=\left(\bar{I}_{1}+\bar{I}_{2}+\bar{I}_{4}\right)\left(\ddot{W}_{-}-\mu^{2} \ddot{W}_{, \xi \xi}\right)
\end{array}
$$

The related edge conditions can also be adjusted in the dimensionless form:

- for a clamped-clamped (C-C) three-layred nanobeam

$$
U_{1}=U_{2}=U_{3}=W=\phi^{*}=0 \quad \text { at } \quad \xi=0,1
$$

— for a simply supported-simply supported (SS-SS) three-layred nanobeam

$$
U_{1}=U_{2}=U_{3}=W=\bar{M}_{x}^{(2)}=0 \quad \text { at } \quad \xi=0,1
$$

— for a clamped-simply supported (C-SS) three-layred nanobeam

$$
\begin{array}{ll}
U_{1}=U_{2}=U_{3}=W=\phi^{*}=0 & \text { at } \quad \xi=0 \\
U_{1}=U_{2}=U_{3}=W=\bar{M}_{x}^{(2)}=0 & \text { at } \quad \xi=1
\end{array}
$$

\section{Modal discretization}

In this part, a reduced order method is used to analyze the behavior of the three-layered nanobeam incorporating the mid-layer shear effect. The derivative terms, in governing equations Eq. (3.2) and related boundary conditions Eqs. (3.3)-(3.5) are discretized by using the Generalized Differential Quadrature Method (GDQM) in order to determine natural frequencies and deflection mode shapes of the three-layred nanobeam. GDQM's main concept is to consider the derivative of a function at a chosen point as a linear weighted sum of the function values at all 
of the surrounding sample points in the corresponding domain (Ke and Wang, 2012). Hence, $U_{1}$, $U_{2}, U_{3}, W, \phi^{*}$ and their $k$-th derivatives regarding $\xi$ can be expressed as

$$
\begin{aligned}
& \left\{U_{1}, U_{2}, U_{3}, W, \phi^{*}\right\}= \\
& \quad \sum_{m=1}^{N} l_{m}(\xi)\left\{U_{1 m}\left(\xi_{m}, t\right), U_{2 m}\left(\xi_{m}, t\right), U_{3 m}\left(\xi_{m}, t\right), W_{m}\left(\xi_{m}, t\right), \phi_{m}^{*}\left(\xi_{m}, t\right)\right\}
\end{aligned}
$$

and

$$
\begin{aligned}
& \left.\frac{\partial^{k}}{\partial \xi^{k}}\left\{U_{1}, U_{2}, U_{3}, W, \phi^{*}\right\}\right|_{\xi=\xi_{i}} \\
& \quad=\sum_{m=1}^{N} C_{i m}^{(k)}\left\{U_{1 m}\left(\xi_{m}, t\right), U_{2 m}\left(\xi_{m}, t\right), U_{3 m}\left(\xi_{m}, t\right), W_{m}\left(\xi_{m}, t\right), \phi_{m}^{*}\left(\xi_{m}, t\right)\right\}
\end{aligned}
$$

where $N$ is the number of grid points dispersed along the beam axis, the nanobeam deflection at the Chebyshev-Gauss-Lobatto grid points $\xi_{i}$ (Ke and Wang, 2012) is given by

$$
\zeta_{i}=\frac{1}{2}\left[1-\cos \frac{\pi(i-1)}{N-1}\right] \quad i=1,2, \ldots, N
$$

The Lagrange interpolation polynomials $l_{m}(\xi)$ are expressed as

$$
l_{m}(\xi)=\frac{R(\xi)}{\left(\xi-\xi_{m}\right) R^{(1)}(\xi)} \quad R(\xi)=\prod_{m=1}^{N}\left(\xi-\xi_{m}\right) \quad R^{(1)}(\xi)=\prod_{m=1, m \neq i}^{N}\left(\xi_{i}-\xi_{m}\right)
$$

and $C_{i m}^{(k)}$ are the weighting coefficients of the $k$-th order differentiation, which can be determined by employing a set of recurrence formulae through the following equations

$$
\left[D_{\xi}^{(k)}\right]_{i j}=C_{i j}^{(k)}= \begin{cases}\frac{R\left(I_{x}\right]_{i j}}{\frac{R\left(\xi_{i}\right)}{\left(\xi_{i}-\xi_{m}\right) R\left(\xi_{m}\right)}} & i \neq m \wedge i, m=1, \ldots, N \wedge k=1 \\ k\left(C_{i m}^{(1)} C_{i i}^{(k-1)}-\frac{C_{i m}^{(k-1)}}{\zeta_{i}-\zeta_{m}}\right) & i \neq m \wedge m=1, \ldots, N \wedge k=2,3, \ldots, N-1 \\ -\sum_{m=1, m \neq i}^{N} C_{i m}^{(k)} & i=m \wedge i, m=1, \ldots, N \wedge k=2,3, \ldots, N-1\end{cases}
$$

where $\mathbf{I}_{x}$ is the $N \times N$ identity matrix.

Considering $\mathbf{U}_{1}, \mathbf{U}_{2}, \mathbf{U}_{3}, \mathbf{W}$ and $\phi^{*}$ defined as

$$
\begin{aligned}
& \mathbf{U}_{1}=\left[U_{11}, U_{12}, U_{13}, \ldots, U_{1 N}\right]^{\mathrm{T}} \quad \mathbf{U}_{2}=\left[U_{21}, U_{22}, U_{23}, \ldots, U_{2 N}\right]^{\mathrm{T}} \\
& \mathbf{U}_{3}=\left[U_{31}, U_{32}, U_{33}, \ldots, U_{3 N}\right]^{\mathrm{T}} \quad \mathbf{W}=\left[W_{1}, W_{2}, W_{3}, \ldots, W_{N}\right]^{\mathrm{T}} \\
& \phi^{*}=\left[\phi_{1}^{*}, \phi_{2}^{*}, \phi_{3}^{*}, \ldots, \phi_{N}^{*}\right]^{\mathrm{T}}
\end{aligned}
$$

and

$$
\begin{aligned}
& U_{1 i}=U_{1}\left(\xi_{i}\right) \quad U_{2 i}=U_{2}\left(\xi_{i}\right) \quad U_{3 i}=U_{3}\left(\xi_{i}\right) \\
& W_{i}=W\left(\xi_{i}\right) \quad \phi_{i}^{*}=\phi^{*}\left(\xi_{i}\right)
\end{aligned}
$$

consequently, we obtain discretized governing equations of motion expressed as

$$
\mathbf{M} \ddot{\mathbf{X}}+\mathbf{K X}=\mathbf{0}
$$


where $\mathbf{X}, \mathbf{K}$ and $\mathbf{M}$ denote, respectively, the vector of variables, stiffness matrix and mass matrix defined as

$$
\begin{aligned}
& \mathbf{X}=\left[\begin{array}{lllll}
\mathbf{U}_{1}^{\mathrm{T}} & \mathbf{U}_{2}^{\mathrm{T}} & \mathbf{U}_{3}^{\mathrm{T}} & \mathbf{W}^{\mathrm{T}} & \boldsymbol{\phi}^{* \mathrm{~T}}
\end{array}\right]^{\mathrm{T}} \\
& \mathbf{K}=\left[\begin{array}{ccccc}
\bar{A}_{11} C_{\xi}^{(2)} & 0 & 0 & 0 & 0 \\
0 & \bar{A}_{12} C_{\xi}^{(2)} & 0 & 0 & 0 \\
0 & 0 & \bar{A}_{13} C_{\xi}^{(2)} & 0 & 0 \\
0 & 0 & 0 & \left(\bar{B}_{11}+\bar{B}_{13}\right) C_{\xi}^{(4)}+k_{s} \bar{C}_{12} C_{\xi}^{(2)} & k_{s} \bar{C}_{12} \eta C_{\xi}^{(1)} \\
0 & 0 & 0 & -k_{s} \bar{C}_{12} C_{\xi}^{(1)} & \bar{B}_{12} C_{\xi}^{(2)}-k_{s} \bar{C}_{12} \eta C_{\xi}^{(0)}
\end{array}\right] \\
& \mathbf{M}=\left[\begin{array}{ccccc}
\bar{I}_{1} D_{\xi} & 0 & 0 & 0 & 0 \\
0 & \bar{I}_{1} D_{\xi} & 0 & 0 & 0 \\
0 & 0 & \bar{I}_{4} D_{\xi} & 0 & 0 \\
0 & 0 & 0 & \left(\bar{I}_{1}+\bar{I}_{2}+\bar{I}_{4}\right) D_{\xi} & 0 \\
0 & 0 & 0 & 0 & \bar{I}_{3} D_{\xi}
\end{array}\right]
\end{aligned}
$$

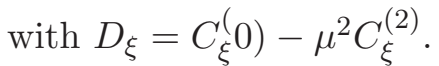

Accordingly, the related edge conditions can be handled in the same way. It follows that for a simply supported-simply supported (SS-SS) three-layred nanobeam incorporating the mid-layer shear effect one obtains

$$
\begin{array}{ll}
U_{11}=U_{21}=U_{31}=W_{1}=\bar{M}_{x 1}^{(2)}=0 & \text { at } \quad \xi=0 \\
U_{1 N}=U_{2 N}=U_{3 N}=W_{N}=\bar{M}_{x N}^{(2)}=0 & \text { at } \quad \xi=1
\end{array}
$$

with

$$
\begin{aligned}
& \bar{M}_{x 1}^{(2)}=\bar{B}_{12} \sum_{m=1}^{N} C_{1 m}^{(1)} \bar{\phi}_{m}+\mu^{2} \bar{I}_{2} \sum_{m=1}^{N} C_{1 m}^{(1)} \ddot{W}_{m} \\
& \bar{M}_{x N}^{(2)}=\bar{B}_{12} \sum_{m=1}^{N} C_{N m}^{(1)} \bar{\phi}_{m}+\mu^{2} \bar{I}_{2} \sum_{m=1}^{N} C_{N m}^{(1)} \ddot{W}_{m}
\end{aligned}
$$

\section{Results and discussion}

In the design of nanostructures, many nano-materials have been used such as carbon nanotubes (CNTs) (Behera et al., 2014) and graphene nanoribbons (GNRs) (Nazemnezhad et al., 2014). The choice of the two nano-materials is based on the superiority of mechanical and electrical properties (Geim, 2009). In fact, these nano-materials have an ultrahigh frequency range up to the terahertz order. In this part, we present numerical results of vibration of the clamped-clamped $(-\mathrm{C})$, simply supported-simply supported (SS-SS) and clamped-simply supported (C-SS) three-layred nanobeam. The three-layred nanobeam is made of bilayer Graphene nanoribbon (BLGNR) with the following material properties $\rho=2260 \mathrm{~kg} \mathrm{~m}^{-3}, G=4.6 \mathrm{GPa}$ and $E=1.06 \mathrm{TPa}$ (Nazemnezhad et al., 2014) and (Hosseini Kordkheili et al., 2013). The effects of the dimensionless nonlocal parameter $\mu$ on vibration frequencies and deflection mode shapes under different boundary conditions are discussed. It is assumed that the length of the nanobeam is $L=14 \mathrm{~nm}$, thicknesses $h_{1}=h_{3}=0.3 \mathrm{~nm}, h_{2}=0.1 \mathrm{~nm}$ and the shear correction factor $k_{s}=0.563$. 


\subsection{Comparison and convergence studies}

In this Section, behavior of the three-layered nanobeam incorporating the mid-layer shear effect with different end supports are analysed. Table 1 presents the fundamental $\omega_{1}[\mathrm{THz}]$ of the three-layred nanobeam with a different number of elements $N$ used for computing the GDQM with $\mu=0.2$. It can be seen that the numerical values get similar to each other as $N$ increases, and those with $N=18$ and 20 are similar for different boundary conditions C-C, SS-SS and C-SS. Therefore, $N=18$ is employed in all subsequent computing.

Table 1. Fundamental frequency $\omega_{1}[\mathrm{THz}]$ of the C-C, SS-SS and C-SS three-layred nanobeam with different $N$

\begin{tabular}{|c|c|c|c|}
\hline$N$ & C-C & SS-SS & C-SS \\
\hline \hline 8 & 0.93886 & 0.63522 & 0.71865 \\
\hline 10 & 0.85984 & 0.41472 & 0.60103 \\
\hline 14 & 0.86111 & 0.43731 & 0.60156 \\
\hline 18 & 0.86117 & 0.45077 & 0.60228 \\
\hline 20 & 0.86117 & 0.45077 & 0.60228 \\
\hline
\end{tabular}

Until now, no theoretical simulations, experimental observations and molecular dynamic results on nonlocal three-layred nanobeams are reported that we can examine and compare the present work against. Hence, in order to ensure efficiency and validity of the proposed model, we consider the two following cases.

In fact, if we decrease thickness of the central layer of the beam comparing to the upper and lower layers thicknesses, the shear effect is neglected and the present model can be directly reduced to the nonlocal Euler-Bernoulli beam model.

Further, if we increase thickness of the central layer of the beam comparing to the upper and lower layers thicknesses, the shear effect is prevalent, so that the present model can be considered as the nonlocal Timoshenko beam model.

Moreover, Wang et al. (2007) analytically analyzed free vibration of an elastic nanobeam based on the nonlocal theory. Tables 2 and 3 give non-dimensional linear frequencies of single-walled carbon nanotubes based on respectively the nonlocal Euler-Bernouilli and Timoshenko beam model. The analytical results provided by Wang et al. (2007) are also given for comparison. Parameters used in this example are taken as (Wang et al., 2007): diameter $d=0.678 \mathrm{~nm}$, Young's modulus $E=5.5 \mathrm{TPa}$, Poisson's ratio $\nu=0.19$, length of beam $L=10 \mathrm{~d}$ and shear correction factor $k_{s}=0.563$.

Solutions obtained by the proposed model of three-layered nanobeams with the shear effect incorporated in the mid-layer based on nonlocal elasticity theory are in good agreement with the analytical results given by Wang et al. (2007) using the nonlocal Euler-Bernoulli and Timoshenko beam theory as well.

\subsection{The proposed nanobeam vibration analysis}

The effect of the scaling parameter $\mu$ on the first four natural frequencies $\omega_{1}-\omega_{4}[\mathrm{THz}]$ of the three layred nanobeam incorporating the mid-layer shear effect for different boundary conditions is presented in Fig. 2. It should be mentioned that the nonlocal parameter $\mu=0$ corresponds to classical nanobeams without the nonlocal effect.

It can be clearly noticed that the nonlocal parameter has a marked effect on the natural frequencies of the three layred nanobeam incorporating the mid-layer shear effect for different edge conditions. Indeed, an increase in the nonlocal parameter leads to a decrease in the natural frequencies. This reduction is more manifested when we consider higher vibration modes. The reduction can be explained by the fact that the nonlocal model may be seen as atoms linked by 
Table 2. Dimensionless frequency of single-walled carbon nanotubes based on the nonlocal Euler Bernoulli beam model considering $h_{1}=h_{3}=0.3 \mathrm{~nm}$ and $h_{2}=0.078 \mathrm{~nm}$ for different boundary conditions

\begin{tabular}{|c|c|c|c|c|}
\hline \multirow{2}{*}{$\begin{array}{c}\text { Frequency } \\
\text { parameter }\end{array}$} & $\begin{array}{c}|c| \\
\text { Wang } \text { et al. } \\
(2007)\end{array}$ & Present & $\begin{array}{c}\text { Wang et al. } \\
(2007)\end{array}$ & Present \\
\hline \hline \multicolumn{5}{|c|}{ C-C } \\
\hline 1 & 4.5945 & 4.4958 & 3.9184 & 3.7276 \\
\hline 2 & 7.1402 & 7.1803 & 5.1963 & 5.2607 \\
\hline 3 & 9.2583 & 9.3447 & 6.2317 & 6.1826 \\
\hline 4 & 11.016 & 11.157 & 7.0482 & 7.1145 \\
\hline \multicolumn{5}{|c|}{ SS-SS } \\
\hline 1 & 3.0685 & 3.2293 & 2.6800 & 2.7139 \\
\hline 2 & 5.7817 & 5.4906 & 4.3013 & 4.3293 \\
\hline 3 & 8.0400 & 8.1050 & 5.4423 & 5.5188 \\
\hline 4 & 9.9162 & 10.072 & 6.3630 & 6.4189 \\
\hline \multicolumn{5}{|c|}{ C-SS } \\
\hline 1 & 3.8209 & 3.7139 & 3.2828 & 3.3506 \\
\hline 2 & 6.4649 & 6.4293 & 4.7668 & 4.6857 \\
\hline 3 & 8.6517 & 8.6378 & 5.4423 & 5.4614 \\
\hline 4 & 10.469 & 10.521 & 6.3630 & 6.4952 \\
\hline
\end{tabular}

Table 3. Dimensionless frequency of single-walled carbon nanotubes based on the nonlocal Timoshenko beam model considering $h_{1}=h_{3}=0.039 \mathrm{~nm}$ and $h_{2}=0.6 \mathrm{~nm}$ for different boundary conditions

\begin{tabular}{|c|c|c|c|c|}
\hline \multirow{2}{*}{$\begin{array}{c}\text { Frequency } \\
\text { parameter }\end{array}$} & $\begin{array}{c}|c| \\
\text { Wang et al. } \\
(2007)\end{array}$ & Present & $\begin{array}{c}\text { Wang et al. } \\
(2007)\end{array}$ & Present \\
\hline \hline \multicolumn{5}{|c|}{ C-C } \\
\hline 1 & 4.3026 & 4.2512 & 3.2420 & 3.3238 \\
\hline 2 & 6.3507 & 6.4276 & 3.9940 & 4.1702 \\
\hline 3 & 8.1969 & 7.9274 & 4.4769 & 4.4708 \\
\hline 4 & 9.5447 & 9.1456 & 5.1131 & 4.9152 \\
\hline \multicolumn{5}{|c|}{ SS-SS } \\
\hline 1 & 3.0243 & 3.1423 & 2.2867 & 2.4693 \\
\hline 2 & 5.5304 & 5.7235 & 3.4037 & 3.2657 \\
\hline 3 & 7.4699 & 7.2662 & 4.1644 & 4.0209 \\
\hline 4 & 8.9874 & 8.6490 & 4.7436 & 4.5083 \\
\hline \multicolumn{5}{|c|}{ C-SS } \\
\hline 1 & 3.6939 & 2.9972 & 2.7471 & 2.9446 \\
\hline 2 & 6.0348 & 6.3202 & 3.7312 & 3.8269 \\
\hline 3 & 7.8456 & 7.5816 & 4.1644 & 4.2341 \\
\hline 4 & 9.2751 & 8.8744 & 4.7436 & 4.6686 \\
\hline
\end{tabular}

elastic springs while in the case of a local continuum model, the spring is constant and supposed to take the infinite value. Consequently, the presence of the nonlocal effect tends to decrease the stiffness of nanostructures and, hence, decreases the values of frequencies (Reddy 2007; Wang et al., 2007). 
(a)

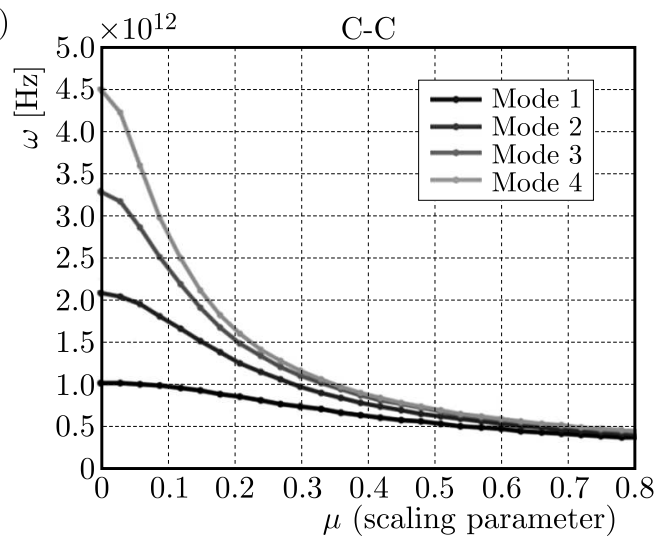

(b)

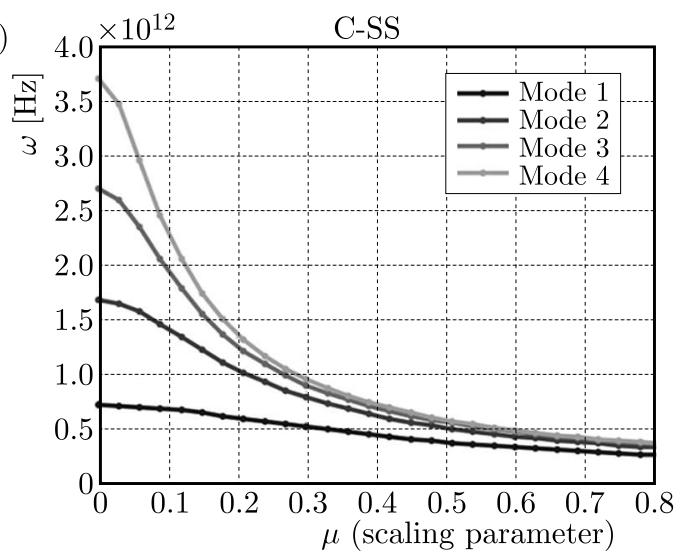

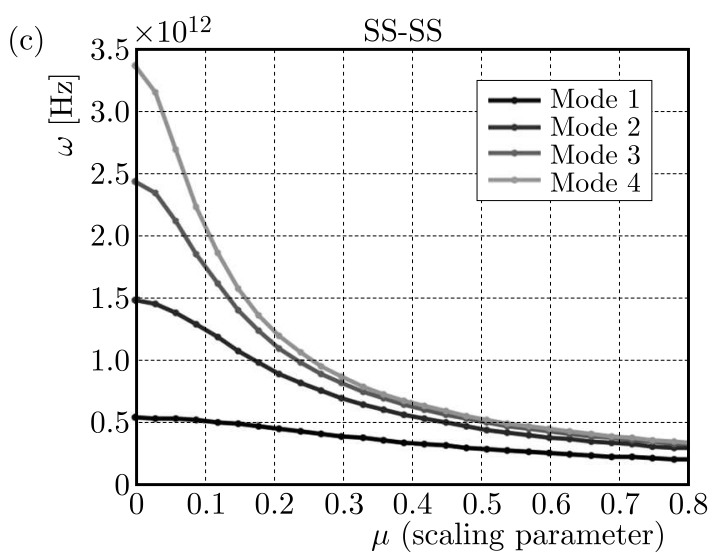

Fig. 2. Effect of the scaling parameter on the first four natural frequencies of three-layered nanobeams for different boundary conditions: (a) C-C, (b) SS-SS, (c) C-SS

(a)

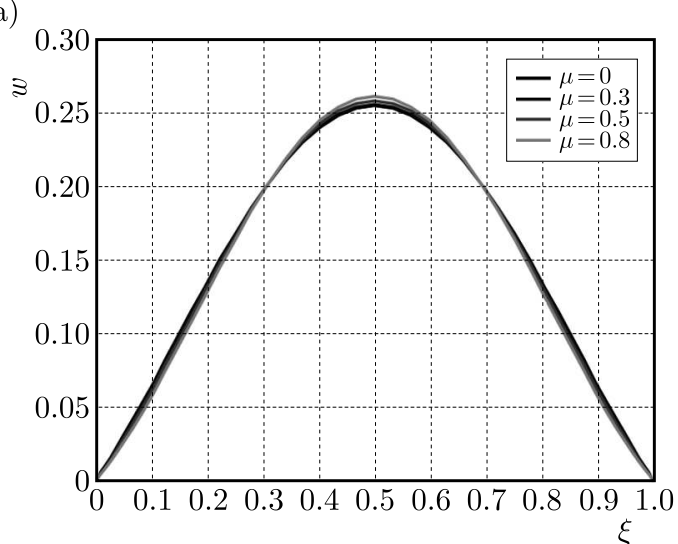

(b)

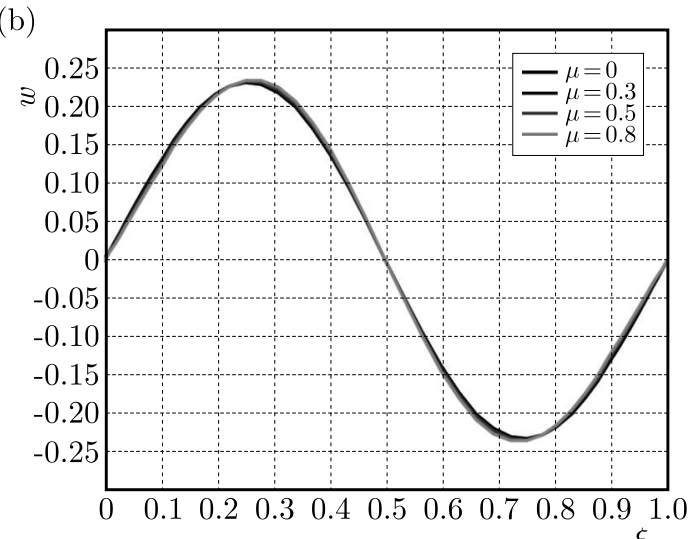

(d)

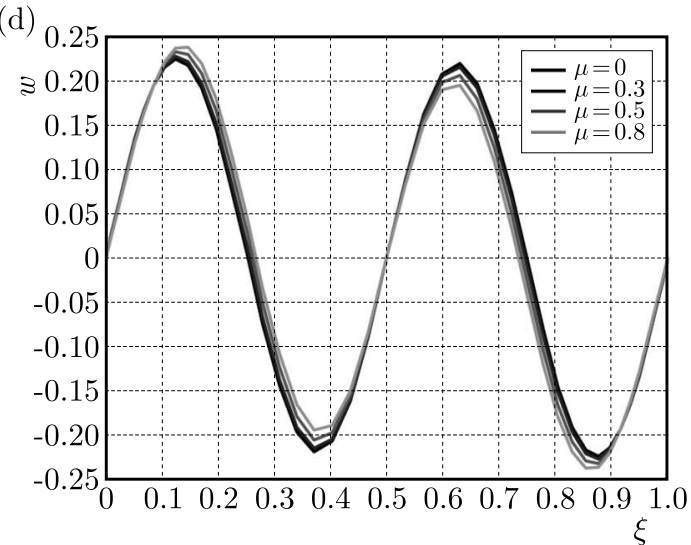

(c)

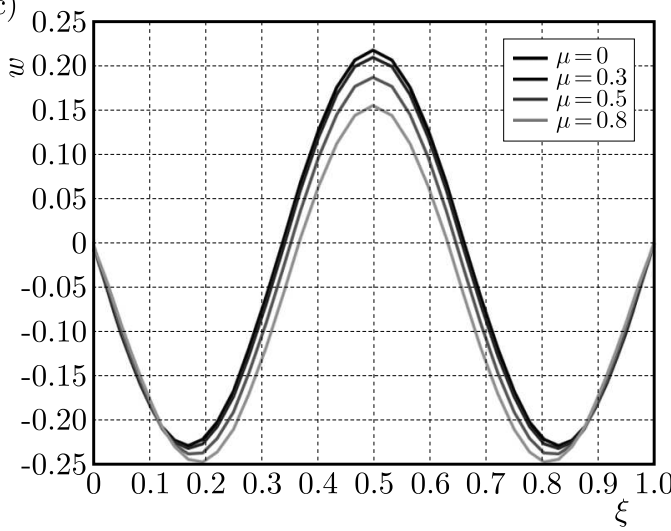


(a)

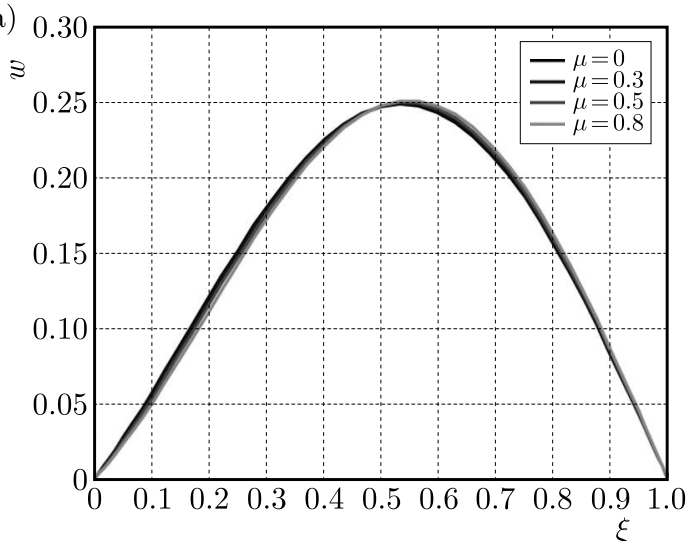

(c)

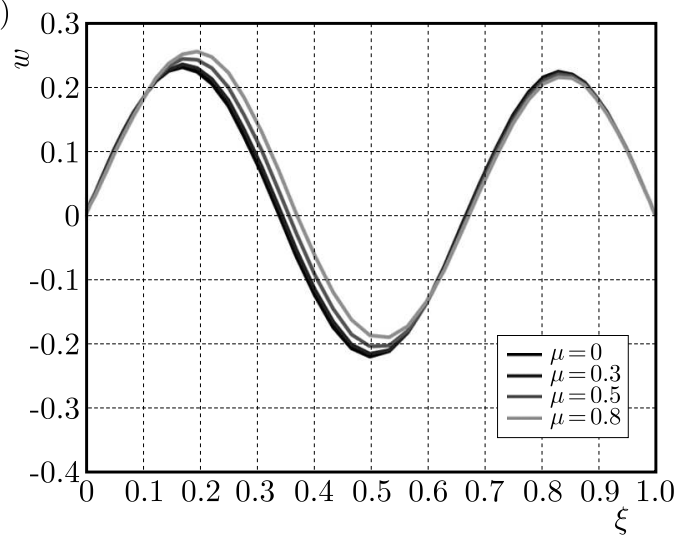

(b)

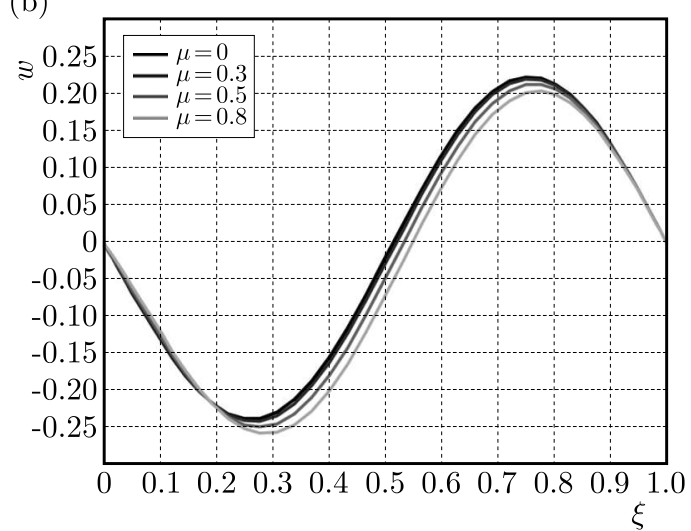

(d)

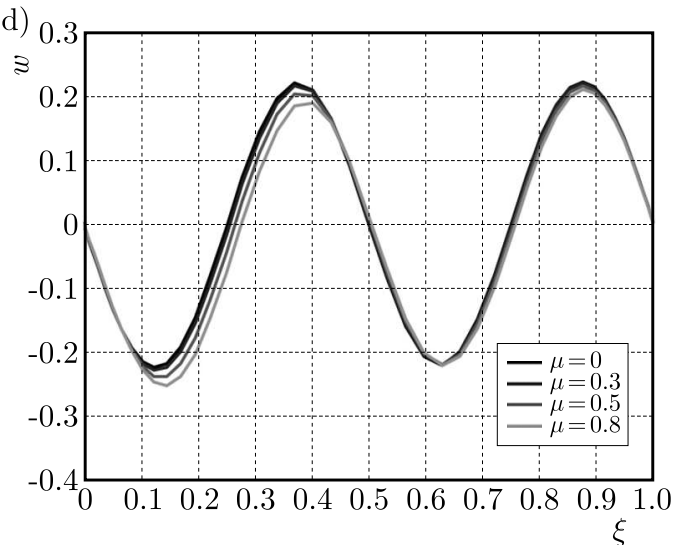

Fig. 4. Effect of the scaling parameter on the first four deflection shapes for C-SS three layered nanobeams: (a) first deflection, (b) second deflection, (c) third deflection and (d) fourth deflection

The C-C nanobeam has higher natural frequencies while the SS-SS has lower natural frequencies since the end support is stronger for the C-C nanobeam and weaker for the SS-SS nanobeam.

Sometimes, the knowledge of higher modes is necessary before finalizing the design of an engineering system. Hence, the first four deflections of nonlocal C-C and C-SS of the proposed three-layred nanobeam are shown respectively in Figs. 3 and 4 for different scaling parameters.

It can be noticed that the deflection mode shapes are affected by an increase in the nonlocal parameter. Deflection graphs of nonlocal C-C and C-SS three-layred nanobeams incorporating the mid-layer shear effect are plotted in this study for different scaling effect parameters to be useful for benchmarking. In fact, by understanding the modes of vibration, we can design structures better in accordance with the need.

\section{Conclusion}

This paper investigates free vibration of three-layered nanobeams incorporating the mid-layer shear effect based on the nonlocal theory, Euler-Bernoulli and Timoshenko beam theories. The GDQM is employed to obtain natural frequencies and deflection mode shapes of the three-layered nanobeams incorporating the mid-layer shear effect with different end supports. Effects of the scaling parameter on vibration characteristics of the proposed nanobeams model are discussed. The results show that an increase in the nonlocal parameter leads to a decrease in the natural frequencies, and the nonlocal parameter nanobeam has a distinguished effect on the mode shapes for the C-C and C-SS nanobeams, but has a less effect on the mode shapes for the SS-SS of the 
proposed nanobeam. Numerical solutions presented herein may be useful to design MEMS and NEMS devices.

\section{References}

1. Ansari R., Gholami R., 2016, Size-dependent nonlinear vibrations of first-order shear deformable magneto-electro-thermo elastic nanoplates based on the nonlocal elasticity theory, International Journal of Applied Mechanics, 8, 4, 1650053-1650086

2. Bauer S., Pittrof A., Tsuchiya H., Schmuki P., 2011, Size-effects in TiO2 nanotubes: Diameter dependent anatase/rutile stabilization, Electrochemistry Communications, 6, 538-541

3. Behera L., Chakraverty S., 2014, Free vibration of Euler and Timoshenko nanobeams using boundary characteristic orthogonal polynomials, Applied Nanoscience, 3, 347-358

4. Eltaher M.A., Emam S.A., Mahmoud F.F., 2013, Static and stability analysis of nonlocal functionally graded nanobeams, Composite Structures, 96, 82-88

5. ERINGEn A.C., 1972, Nonlocal polar elastic continua, International Journal of Engineering Science, 10, 1, 1-16

6. Geim A.K., 2009, Graphene: Status and Prospects, Manchester Centre for Mesoscience and Nanotechnology, University of Manchester, Oxford Road M13 9PL, Manchester, UK, 324, 1-8

7. He J.H., 1999, Homotopy perturbation technique, Computer Methods in Applied Mechanics and Engineering, 178, 257-262

8. Hosseini Kordkheili S.A., SANi H., 2013, Mechanical properties of double-layered graphene sheets, Computational Materials Science, 69, 335-343

9. Hung E.S., Senturia S.D., 1999, Extending the travel range of analog-tuned electrostatic actuators, Journal of Microelectromechanics Systems, 8, 497-505

10. Ke L.L., WANG Y.S., 2012, Thermoelectric-mechanical vibration of piezoelectric nanobeams based on nonlocal theory, Smart Materials and Structures, 21, 1-12

11. Ke L.L., WANG Y.S., 2014, Free vibration of size-dependent magneto-electro-elastic nanobeams based on the nonlocal theory, Physica E, 63, 52-61

12. Koiter W.T., 1964, Couple-stresses in the theory of elasticity: I and II, Koninklijke Nederlandse Akademie van Wetenschappen (Royal Netherlands Academy of Arts and Sciences), 67, 17-44

13. Lam D.C.C., Yang F., Chong A.C.M., Wang J., Tong P., 2003, Experiments and theory in strain gradient elasticity, Journal of Mechanics and Physics of Solids, 51, 1477-1508

14. Li C., Lim C.W., Yu J.L., 2011, Dynamics and stability of transverse vibrations of nonlocal nanobeams with a variable axial load, Smart Materials and Structures, 20

15. Li X., Bhushan B., Takashima K., Baek C.W., Kim Y.K., 2003, Mechanical characterization of micro/nanoscale structures for MEMS/NEMS applications using nanoindentation techniques, Ultramicroscopy, 97, 481-494

16. Lim C.W., 2010, On the truth of nanoscale for nanobeams based on nonlocal elastic stress field theory: equilibrium, governing equation and static deflection, Applied Mathematics and Mechanics, $1,37-54$

17. Minduin R.D., 1963, Influence of couple-stresses on stress concentrations, Experimental Mechanics, $3,1-7$

18. Minduin R.D., 1965, Second gradient of strain and surface-tension in linear elasticity, International Journal of Solids and Structures, 1, 217-238

19. Moser Y., Gijs M.A.M., 2007, Miniaturized flexible temperature sensor, Journal of Microelectromechanical Systems, 16, 1349-1354 
20. Mousavi T., Bornassi S., Haddadpour H., 2013, The effect of small scale on the pull-in instability of nano-switches using DQM, International Journal of Solids and Structures, 50, 1193-1202

21. Najar F., Nayfeh A.H., Abdel-Rahman E.M., Choura S., El-Borgi S., 2010, Global stability of microbeam-based electrostatic microactuators, Journal of Vibration and Control, 16, 721-748

22. Nazemnezhad R., Hosseini-Hashemi S., 2014, Free vibration analysis of multi-layer graphene nanoribbons incorporating interlayer shear effect via molecular dynamics simulations and nonlocal elasticity, Physics Letters A, 44, 3225-3232

23. NAzemnezhad R., Zare M., 2016, Nonlocal Reddy beam model for free vibration analysis of multilayer nanoribbons incorporating interlayer shear effect, European Journal of Mechanics A/Solids, 55, 234-242

24. Nilsson J., Neto A.C., Guinea F., Peres N., 2008, Electronic properties of bilayer and multilayer graphene, Physical Review B, 78, 4, 405-434

25. Peddieson J., Buchanan G.R., McNitt R.P., 2003, Application of nonlocal continuum models to nanotechnology, International Journal of Engineering Science, 41, 305-312

26. Pei J., Tian F., Thundat T., 2004, Glucose biosensor based on the microcantilever, Analytical Chemistry, 76, 292-297

27. REDDy J.N., 2007, Nonlocal theories for bending, buckling and vibration of beams, International Journal of Engineering Science, 45, 288-307

28. REDDY J.N., 2010, Nonlocal nonlinear formulations for bending of classical and shear deformation theories of beams and plates, International Journal of Engineering Science, 48, 1507-1518

29. Reddy J.N., El-Borgi S., 2014, Eringen's nonlocal theories of beams accounting for moderate rotations, International Journal of Engineering Science, 82, 159-177

30. Roque C.M.C., Ferreira A.J.M., Reddy J.N., 2011, Analysis of Timoshenko nanobeams with a nonlocal formulation and meshless method, International Journal of Engineering Science, 49, 976-984

31. Shkel A.M., 2006, Type I and Type II micromachined vibratory gyroscopes, Proceedings of the IEEE/Institute of Navigation Plans, San Diego, CA, 586-593

32. SimseK M., 2014, Large amplitude free vibration of nanobeams with various boundary conditions based on nonlocal elasticity theory, Composites: Part B, 56, 621-628

33. Thai H.T., 2012, A nonlocal beam theory for bending, buckling, and vibration of nanobeams, International Journal of Engineering Science, 52, 56-64

34. Toupin R.A., 1964, Theories of elasticity with couple-stress, Archive for Rational Mechanics and Analysis, 17, 85-112

35. WANG Q., Wang C.M., 2007, The constitutive relation and small scale parameter of nonlocal continuum mechanics for modelling carbon nanotubes, Nanotechnology, 18, 7 